\title{
Loss of Data
}

National Cancer Institute

\section{Source}

National Cancer Institute. Loss of Data. NCI Thesaurus. Code C63257.

Event in which data is unintentionally permanently or temporarily lost, deleted, corrupted, or overwritten. 